\title{
Anti-PD-L1 immune checkpoint inhibitors in combination with etoposide and platinum for extensive-stage small cell lung cancer: a case report
}

\author{
Lei Xue $^{1 \#}$, Baishen Chen ${ }^{2 \#}$, Junshuang Lin ${ }^{1}$, Jiangzhou Peng ${ }^{1}$ \\ ${ }^{1}$ Department of Thoracic Surgery, The Third Affiliated Hospital of Southern Medical University, Guangzhou, China; ${ }^{2}$ Department of Thoracic \\ Surgery, Sun Yat-sen Memorial Hospital, Sun Yat-sen University, Guangzhou, China \\ \#These authors contributed equally to this work. \\ Correspondence to: Jiangzhou Peng. Department of Thoracic Surgery, The Third Affiliated Hospital of Southern Medical University, Guangzhou \\ 510630, China. Email: academycn@foxmail.com.
}

\begin{abstract}
The conventional etoposide-platinum (EP) regimen and adjuvant radiotherapy remain the gold-standard treatment for small cell lung cancer (SCLC). However, most patients already have multiple metastases when they are first diagnosed with SCLC. The objective response rate (ORR) and 1-year survival rate are low in these patients despite active radiotherapy and chemotherapy. SCLC is oncologically featured by the high tumor mutational burden (TMB) of multiple genes, which makes immunotherapy a possible new treatment strategy for SCLC. New data from the IMpower133 and CASPIAN trials will shed new light on the treatment of SCLC. In 2020, the results from the phase 3 CASPIAN trial have already suggested that programmed cell death-ligand 1 (PD-L1) inhibitors may represent breakthroughs in the management of SCLC. Here, we report a patient with extensive-stage SCLC (ES-SCLC) treated with first-line antiPD-L1 immune checkpoint inhibitor (PD-L1 inhibitor) (i.e., durvalumab) combined with the EP regimen for 6 cycles. The patient consistently achieved partial response (PR) [nearly complete response (CR)], and no immune-related adverse events were noted during this period. The Karnofsky performance status (PS) score maintained at 1-2 points. We further review the history of SCLC treatment and elucidate the role of combination with immunotherapy in treating SCLC in the coming years.
\end{abstract}

Keywords: Small cell lung cancer (SCLC); programmed death-1 (PD-1); programmed cell death-ligand 1 (PD-L1); tumor mutation burden (TMB); PD-L1 expression

Submitted Nov 12, 2020. Accepted for publication Jan 11, 2021.

doi: 10.21037/apm-20-2574

View this article at: http://dx.doi.org/10.21037/apm-20-2574

\section{Introduction}

Lung cancer is the leading cause of cancer death worldwide. Small cell lung cancer (SCLC) is one of the most aggressive and lethal types, accounting for approximately $15 \%$ of patients diagnosed with lung cancer (1). SCLC has a poor prognosis due its high rates of tumor cell proliferation, metastasis, and resistance to current treatments (2). Most patients already have extensive-stage SCLC (ES-SCLC) at diagnosis, and only a small proportion of the tumors are surgically resectable. Few advances have been made in the management of SCLC, and the etoposide-platinum (EP) regimen combined with adjuvant radiotherapy remains the gold-standard treatment. SCLC has complex gene mutations, which make it impossible to develop specific targeted drugs. Pathologically, however, the tumor is featured by high tumor mutational burden (TMB) of multiple genes, suggesting that DNA errors caused by cell replication and mutations continue to accumulate, and cannot be recognized or eliminated by the immune system in a timely manner. The lack of immune surveillance may be induced by tumors. Programmed death-1 (PD1)/programmed cell death-ligand 1 (PD-L1) is one of 


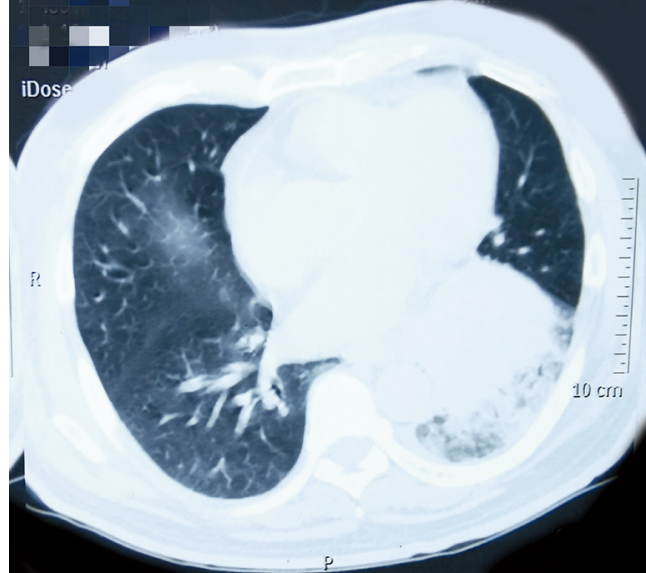

Figure 1 Chest CT before treatment (March, 29, 2020).

the negative regulatory pathways in the human body. In the tumor microenvironment, it is a common for the expression of PD-L1 protein on the surface of tumor cells to be upregulated; after the PD-L1 protein binds to the PD-1 receptor on the surface of lymphocytes, it initiates negative immune feedback that prevents $T$ cells from recognizing cancer cells. The accumulated errors in tumor cells and mutations finally result in a silenced immune response. Durvalumab is a PD-L1 inhibitor that can bind to the PD-L1 receptor on the surface of tumor cells in the tumor microenvironment, which allows $\mathrm{T}$ cells to be excluded from the tumor-induced immune silencing; accordingly, the normal immune surveillance mechanism is reactivated to exert its functions in recognizing and killing tumor cells.

We present the following case in accordance with the CARE reporting checklist (available at http://dx.doi. org/10.21037/apm-20-2574).

\section{Case presentation}

A 68-year-old male patient was admitted due to "chest tightness and shortness of breath for 2 months" on March 25, 2020, with no special medical, family, and psycho-social history. Chest positron emission tomography-computed tomography (PET-CT) on March 29, 2020, revealed a huge space-occupying mass about $8 \mathrm{~cm} \times 9 \mathrm{~cm} \times 6.5 \mathrm{~cm}$ in size in the left lower lobe of the lung, along with obstructive pneumonia. The possibility of central bronchogenic carcinoma was considered (Figures 1 and 2). The findings of bronchoscopic pathology are shown in Figures 3-5. The microscopic morphologies and immunolabeling findings

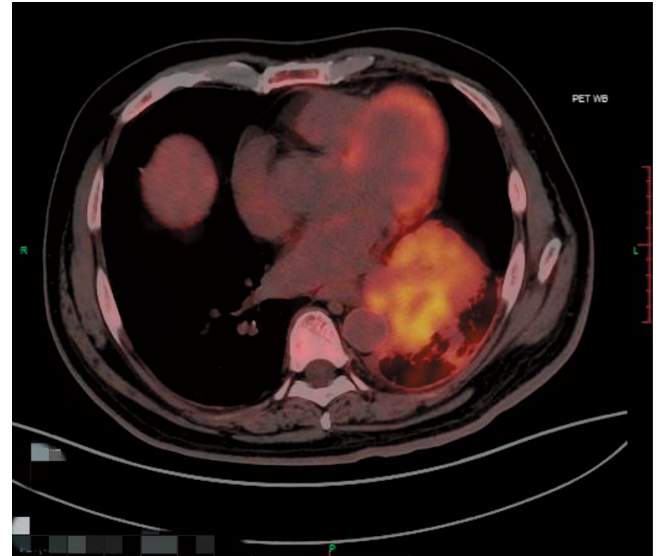

Figure 2 PET-CT before treatment (April, 10, 2020). PET-CT, positron emission tomography-computed tomography.

supported the diagnosis of SCLC. The patient further received systemic assessments. In particular, PET-CT was performed. The possible diagnoses included SCLC with hilar and mediastinal lymph node metastases and bone metastasis. The clinical stage was $\mathrm{cT}_{4} \mathrm{~N}_{2} \mathrm{M}_{1 \mathrm{c}}$. He began to receive immunotherapy combined with chemotherapy on April 6, 2020. The specific protocol was 6 cycles of durvalumab $1,000 \mathrm{mg}(10 \mathrm{mg}$ per $\mathrm{kg}) \mathrm{q} 3 \mathrm{w}$ combined with an EP regimen. The treatment went smoothly. Neither adverse reactions nor immune-related side effects were noted. The Karnofsky performance status (PS) score maintained at 1-2 points. His quality of life was satisfactory. During the entire clinical follow-up period, the solid lung tumor continued to shrink (Figures 6-11), and the neuron-specific enolase (NSE), a specific marker of SCLC, was completely resolved before the second cycle of treatment (Table 1). The final session of the immunotherapy-chemotherapy combination was given on August 21, 2020. According to the results of the CASPIAN III trial, maintenance treatment (durvalumab monotherapy maintenance, dose $\mathrm{q} 3 \mathrm{w}$ ) was applied beginning from the seventh session. The last imaging examination was performed at the patient's home on October 13, 2020 (before the initiation of the single-drug maintenance treatment), when chest CT revealed a space-occupying lesion sized about $1 \mathrm{~cm} \times 0.7 \mathrm{~cm}$ $\times 0.5 \mathrm{~cm}$ in the basal segment of the left lower lobe, which was markedly smaller than before treatment, indicating the approach to complete response (CR). According to the evaluation criteria, the patient has achieved a partial response (PR).

All procedures performed in studies involving human 


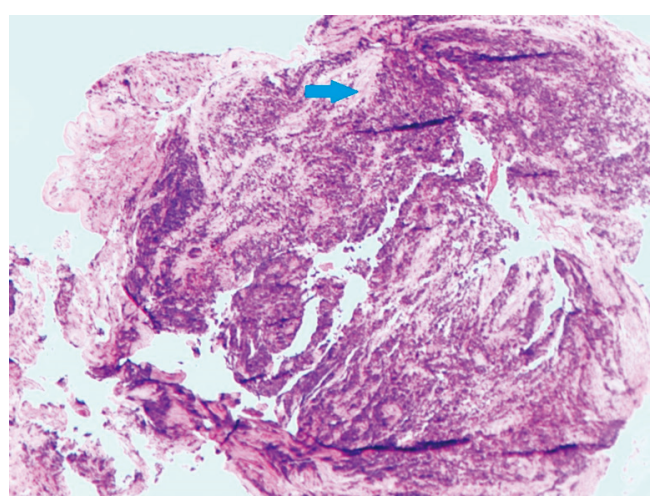

Figure 3 Pathological section ( $\times 40$, HE staining).

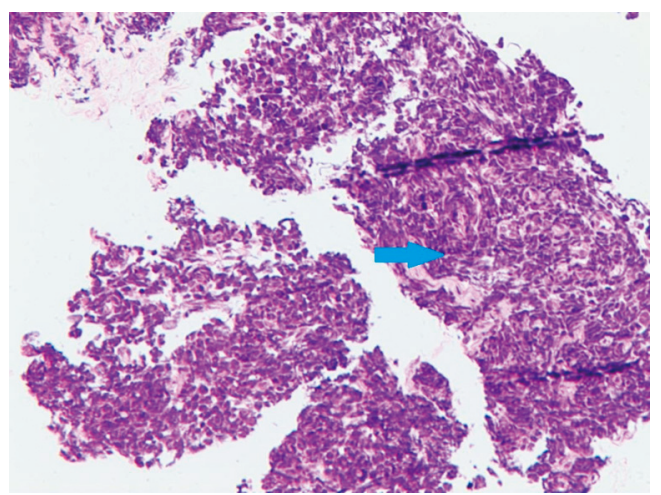

Figure 4 Pathological section $(\times 100$, HE staining).

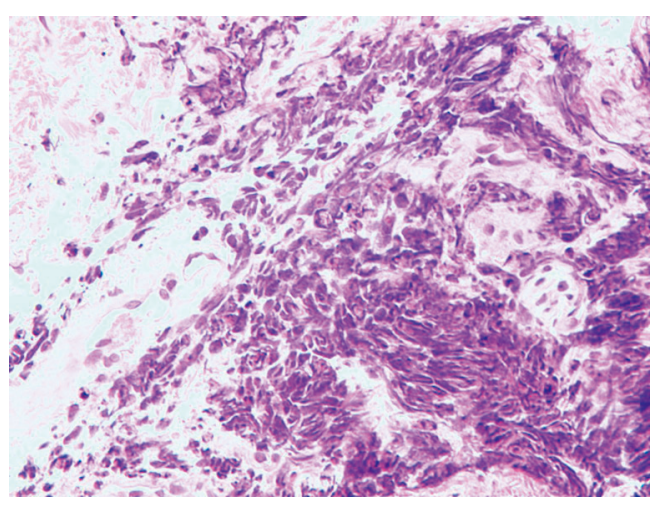

Figure 5 Pathological section $(\times 200$, HE staining).

participants were in accordance with the ethical standards of the institutional and/or national research committee(s) (No. 2019-018) and with the Helsinki Declaration (as revised in 2013). Written informed consent was obtained from the patient.

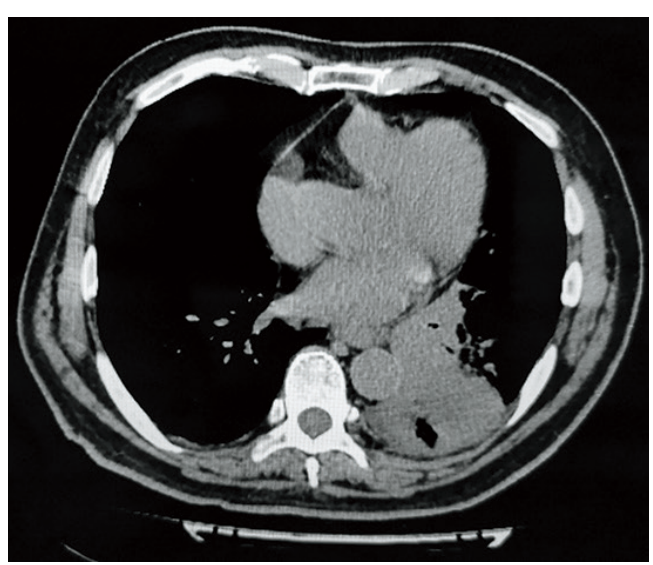

Figure 6 After the first cycle of durvalumab plus EP (May, 19, 2020). EP, etoposide-platinum.

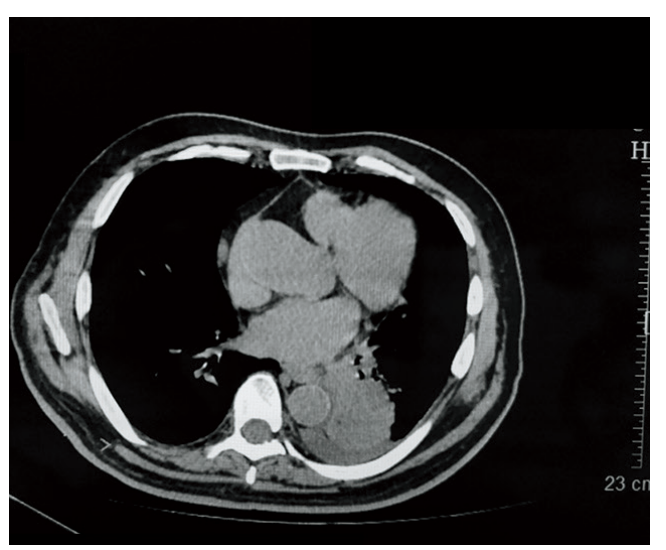

Figure 7 After the second cycle of durvalumab plus EP (June, 10, 2020). EP, etoposide-platinum.

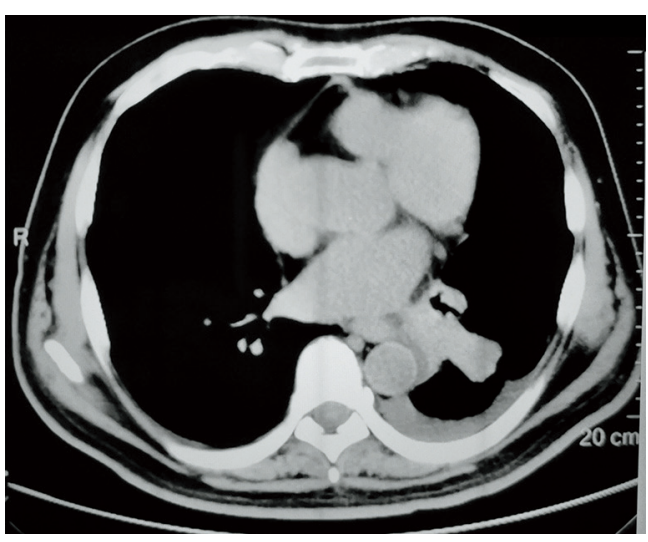

Figure 8 After the third cycle of durvalumab plus EP (June, 30, 2020). EP, etoposide-platinum. 


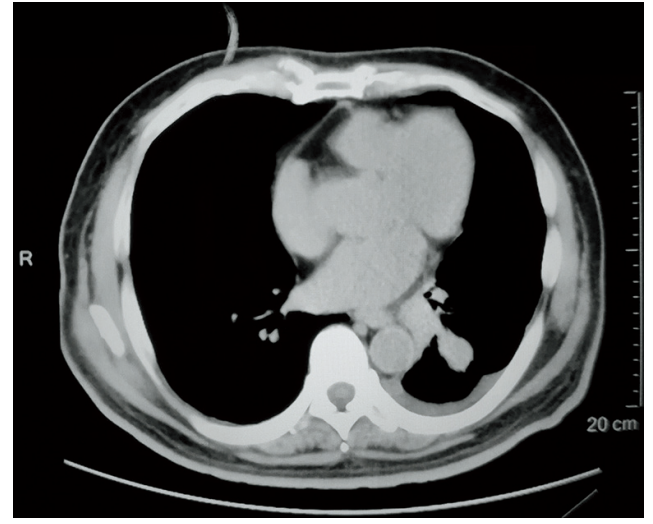

Figure 9 After the fourth cycle of durvalumab plus EP (July, 23, 2020). EP, etoposide-platinum.

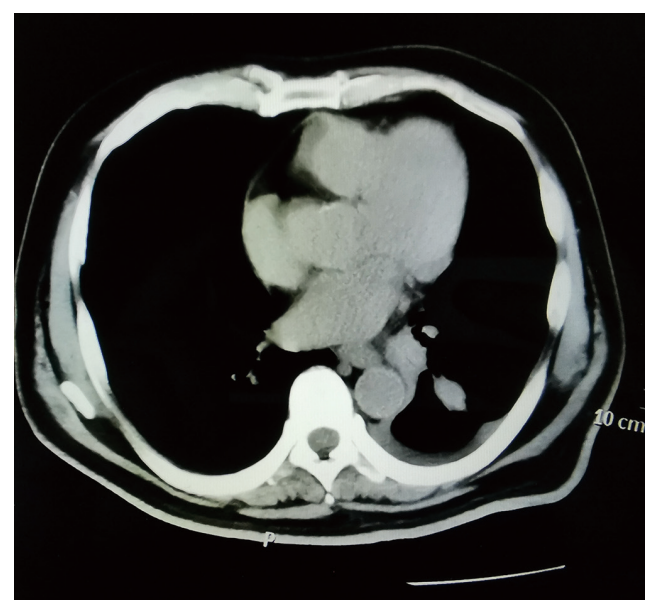

Figure 10 After the fifth cycle of durvalumab plus EP (August, 15, 2020). EP, etoposide-platinum.

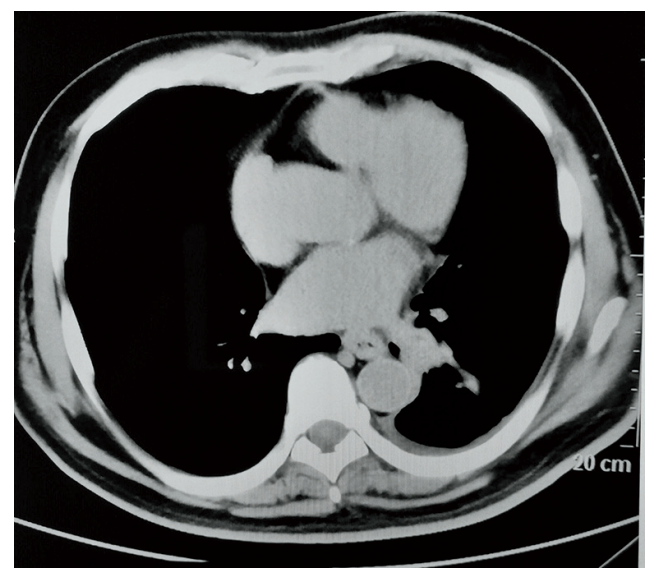

Figure 11 After the sixth cycle of durvalumab plus EP (October, 13, 2020). EP, etoposide-platinum.

\section{Discussion}

While lung cancer is the most fatal cancer worldwide, SCLC is the most dangerous type of lung cancer, accounting for about $10-15 \%$ of lung cancer cases (3). SCLC has the biological characteristics of aggressive neuroendocrine tumors (4) and is featured by early metastasis, frequent recurrence, high proliferation rate, and high prevalence of drug-resistance mutations. Up to $60 \%$ of patients are already at the extensive stage at the time of diagnosis (5).

ES-SCLC is sensitive to initial chemotherapy but is prone to rapid relapse and drug resistance shortly after remission. The duration of disease control (DDC) is short, and the tumor often becomes refractory to chemotherapy after recurrence (6). The vast majority of SCLC patients will progress to clinical death within 2 years after disease relapse and drug resistance (7). A small proportion of limited-stage SCLC cases can be treated by surgery, radiotherapy, chemotherapy, and some other options. However, the therapeutic responses are far from satisfactory as the disease progresses. Only a small number of stage I SCLC patients can benefit significantly from surgery combined with radiotherapy and chemotherapy (8). Most stage II, T1-2N1M0, and T3N0M0 patients have no significant benefit in overall survival (OS) after surgery $(9,10)$, and limited-stage SCLC can easily metastasize to the brain. Its first-line treatment remains an EP regimen combined with prophylactic cranial irradiation (PCI). However, the response rate is low, with a 5 -year survival rate of only about $6 \%$ and an average OS of less than 10 months (11).

Meanwhile, due to the lack of targetable mutations in SCLC (12), few breakthroughs have been made in the targeted therapy of SCLC. Rovalpituzumab tesirine (Rova-T), which showed initial promise, is an antibodydrug conjugate that targets cancer stem cell-related deltalike ligand 3 (DLL3). A preliminary study reported that its disease control rate (DCR) could reach 91\% (13). However, its TRINITY phase II trial showed that, even in DLL3positive patients, the objective response rate (ORR) was only $13.2 \%$, the median OS (mOS) was only 5.8 months, and the 1 -year OS was only $17.5 \%$; notably, $63 \%$ of patients suffered from grade 3 to 5 immune-related adverse events (irAEs) (14). In the TAHOE phase III trial, the Rova-T group was significantly inferior to the topotecan group in terms of OS, which led to the premature cessation of the phase III trial. Ipilimumab, which targets cytotoxic T-lymphocyte antigen-4 (CTLA-4), was also a once 
Table 1 Neuron-specific enolase changes

\begin{tabular}{lccc}
\hline Neuron-specific enolase & April, 9, 2020, initiation & May, 19, 2020, first session & June, 10, 2020, second session \\
\hline NSE (0-0.2 ng/mL) & 70.71 & 12.02 & 8.41 \\
\hline
\end{tabular}

NSE, neuron-specific enolase.

highly anticipated drug. However, its combinations with standard platinum-based chemotherapy regimens as firstline treatment of ES-SCLC did not produce any benefit in progression-free survival (PFS) or OS (15).

As can be seen, despite myriad efforts, there has been no breakthrough in SCLC treatment for nearly 30 years. In 2012, a single-nucleotide polymorphism (SNP) analysis based on 63 SCLS tumor tissue samples showed that the genomic mutation rate in SCLC cells was $7.4 \mathrm{mut} / \mathrm{Mbp}$, suggesting that patients with SCLC have a high TMB (16); however, no specific mutation marker has been found. Thus, SCLC has a uniquely high TMB and lacks any characteristic targetable mutations.

Once there is a problem with the immune surveillance mechanism of SCLC patients, the "damaged cells" cannot be recognized and eliminated by the immune system in time. Correcting the imbalance of the immune system may be a novel solution in the treatment of SCLC.

In 2002, American tumor biologist R.D. Schreiber proposed his now-renowned hypothesis of "cancer immunoediting" to explain the relationship between tumors and the immune system. The occurrence and development of cancer cells in the body constitute a dynamic process in which the immune system interacts with cancer cells. In this process, the immune system reshapes other biological characteristics (such as tumor antigenicity and invasiveness) of tumor cells, which is the so-called "immunoediting", while removing tumor cells. The immunoedited tumor cells become more malignant. These immunologically screened tumor cells induce the silencing of immune system, rendering it incapable of recognizing tumor cells and leading to their uncontrolled growth and spread.

According to the cancer immunoediting theory, the relationship between the immune system and tumors can be divided into three different phases: elimination, equilibration, and escape. In elimination, a screening process occurs in which many tumor cells are destroyed by the immune response, but a portion survive. These screened tumor cell subgroups grow rapidly to form a microenvironment that suppresses immune cells, where the tumor cells release molecules with immunosuppressive functions and induce the immune cell differentiation potentials. For example, these tumor cells may induce the production of CTLA-4-expressing regulatory $\mathrm{T}$ cells; the CD4+CD25+FOXP3+ $\mathrm{T}$ cells in turn induce an upregulation of immunosuppressive functions, and accelerate the depletion of cytotoxic T cells (17). The suppression and exhaustion of the immune system leads to the breakdown of equilibration. As a result, the immune system cannot offer an effective immune response to tumor proliferation and metastasis.

Many studies have investigated the upregulated PDL1 ligand expression on the surface of tumor cells and the binding pathway of immune checkpoint PD-1 receptor on the surface of immune cells. These mechanisms may induce the silencing of lymphocytes against tumor cells $(18,19)$. PD-1/PD-L1 pathway inhibitors disrupt the binding of PD-1 to PD-L1, block negative regulatory signals, and release the inhibited $\mathrm{T}$ cell activity (20), thereby restoring $\mathrm{T}$ cell activity and enhancing immune response. Thus, the dynamic surveillance and balance between the immune system and tumors are restored.

Theoretically, both PD-1 and PD-L1 inhibitors will eventually block the PD-1/PD-L1 pathway, although their ligands are slightly different. However, the KEYNOTE 604 and IMPower133 trials reported that PD-1 inhibitors did not yield better survival outcomes in SCLC patients. However, PD-L1 inhibitors significantly improved OS. The exact mechanisms for this difference remain unclear, but may be explained by the role of PD-L1 in blocking CD80 signaling.

IMPower133 is a phase III, multicenter, double-blind, randomized placebo-controlled study evaluating the role of PD-L1 inhibitors combined with the EP regimen in treating treatment-naïve ES-SCLC patients. This combination was the first new treatment in the past 30 years to demonstrate a better effect than first-line EP chemotherapy in improving prognosis. The updated results of the IMPower133 trial have further shown that patients with ES-SCLC have gained obvious OS benefits. Based on the results of the IMPower133 trial, the U.S. Food and Drug Administration (FDA) has accepted for priority review a Biologics License 
Application (BLA) for PD-L1 inhibitors. In 2019, the National Comprehensive Cancer Network (NCCN) Clinical Practice Guidelines in Oncology Version 1.2019 recommended the use of atezolizumab in combination with the EP regimen in the first-line treatment of adult patients with ES-SCLC. In the same year, the Chinese Society of Clinical Oncology (CSCO) also included atezolizumab combined with EP regimen as the first-line treatment of adult patients with ES-SCLC in its guidelines.

The CASPIAN study is a randomized, open-label, global multicenter phase III clinical trial on the first-line treatment of patients with ES-SCLC. Treat-naïve patients with ESSCLC were enrolled. In February 2020, the CASPIAN phase III trial published its findings, which showed that, compared with chemotherapy alone, durvalumab combined with chemotherapy could significantly improve OS. The median survival was 10.5 months in the chemotherapy-alone group and reached 12.9 months in the durvalumab plus chemotherapy group [hazard ratio $(\mathrm{HR})=0.73, \mathrm{P}=0.0047$ ]; the 12 -month OS rate was $39.8 \%$ in the chemotherapyalone group but was $53.7 \%$ in the combination group; and the ORR was $57.6 \%$ and $67.9 \%$ in the chemotherapy-alone group and combination group, respectively. In addition, the survival rates in these two groups after 18 months of treatment were $24.7 \%$ and $33.9 \%$, respectively. After 24 months of treatment, the rate of sustained remission was $3.9 \%$ and $13.5 \%$ in the chemotherapy-alone group and combination group, respectively $(21,22)$.

The literature indicates that the immunotherapy of SCLC does not depend on the expression level of PDL1, which may be due to the high TMB and imbalanced immune surveillance in SCLC. High TMB means that the abnormal antigens on the surface of tumor cells can be easily recognized by the immune system and become the targets of attack. The uncovering of the unique mechanism of immunotherapy provides new ideas for the treatment of SCLC. The treatment of our current patient used a combination protocol (durvalumab $10 \mathrm{mg} / \mathrm{kg}$ $\mathrm{q} 3 \mathrm{w}+\mathrm{EP}$ regimen), with the NSE being completely resolved before the second treatment cycle. After the third cycle, the patient had achieved PR according to results of imaging assessment, and the time to PR was significantly shorter than that in the traditional EP chemotherapy. The TMB was markedly reduced after the sixth cycle of treatment, and the tumor continued to shrink during the follow-up period. Durvalumab single-agent maintenance therapy may eventually achieve clinical CR. Currently there is an opportunity for radical resection of local tumor.
Determining whether a major pathologic response (MPR) or even a complete pathologic response (CPR) at the cellular level could be achieved requires further examination and treatment efforts. Furthermore, the cumulative effect of PD-1 inhibitors can lead to a high incidence of immune enteritis at late stages; in contrast, no definite drug-related adverse event was noted during the durvalumab treatment. During the durvalumab treatment, the bone metastases were stable, alkaline phosphatase was not abnormally elevated, and there were no bone metastasis-related adverse events (e.g., pathological fractures). The patient has finished 6 cycles of immunotherapy, and surgical resection or immunotherapy for maintenance will be considered. No irAEs have occurred yet. It has been reported that immunotherapy combined with chemotherapy can effectively reduce the occurrence of irAEs in patients with non-small cell lung cancer (NSCLC) (23), and a similar outcome has been seen in our current SCLC patient.

As a major breakthrough in tumor immunology in the past decade, the discovery of the tumor immunosuppressive mechanism has promoted advances in tumor immunotherapy. Initially it was thought that chemotherapy and other cytotoxic treatments would destroy the immune system and cause immunosuppression, thereby hindering the efficacy of immunotherapy. Since then, the view that immunotherapy combined with chemotherapy can increase anti-tumor $\mathrm{T}$ cell response through cross-priming and reduce irAEs has gradually been accepted (24). This phenomenon is especially prominent in the treatment of SCLC. Nevertheless, whether chemotherapy combined with immunotherapy can prolong its tail effect warrants further investigation.

\section{Acknowledgments}

Funding: None.

\section{Footnote}

Reporting Checklist: The authors have completed the CARE reporting checklist. Available at http://dx.doi.org/10.21037/ apm-20-2574

Conflicts of Interest: All authors have completed the ICMJE uniform disclosure form (available at http://dx.doi. org/10.21037/apm-20-2574). The authors have no conflicts of interest to declare.

Ethical Statement: The authors are accountable for all 
aspects of the work in ensuring that questions related to the accuracy or integrity of any part of the work are appropriately investigated and resolved. All procedures performed in studies involving human participants were in accordance with the ethical standards of the institutional and/or national research committee(s) (No. 2019-018) and with the Helsinki Declaration (as revised in 2013). Written informed consent was obtained from the patient.

Open Access Statement: This is an open access article distributed in accordance with the Creative Commons Attribution-NonCommercial-NoDerivs 4.0 International License (CC BY-NC-ND 4.0), which permits the noncommercial replication and distribution of the article with the strict proviso that no changes or edits are made and the original work is properly cited (including links to both the formal publication through the relevant DOI and the license). See: https://creativecommons.org/licenses/bync-nd/4.0/.

\section{References}

1. Siegel RL, Miller KD, Jemal A. Cancer statistics, 2020. CA Cancer J Clin 2020;70:7-30.

2. van Meerbeeck JP, Fennell DA, De Ruysscher DKM. Smallcell lung cancer. Lancet 2011;378:1741-55.

3. Kalemkerian GP, Akerley W, Bogner P, et al. Small cell lung cancer. J Natl Compr Canc Netw 2013;11:78-98.

4. Ouadah Y, Rojas ER, Riordan DP, et al. Rare Pulmonary Neuroendocrine Cells Are Stem Cells Regulated by Rb, p53, and Notch. Cell 2019;179:403-16.e23.

5. Pesch B, Kendzia B, Gustavsson P, et al. Cigarette smoking and lung cancer--relative risk estimates for the major histological types from a pooled analysis of case-control studies. Int J Cancer 2012;131:1210-9.

6. Jalal S, Ansari R, Govindan R, et al. Pemetrexed in second line and beyond small cell lung cancer :a Hoosier Oncology Group phase II study. J Thorac Oncol 2009;4:93-6.

7. Byers LA, Rudin CM. Small cell lung cancer: where do we go from here? Cancer 2015;121:664-72.

8. Gaspar LE, McNamara EJ, Gay EG, et al. Small-cell lung cancer: prognostic factors and changing treatment over 15 years. Clin Lung Cancer 2012;13:115-22.

9. Combs SE, Hancock JG, Boffa DJ, et al. Bolstering the case for lobectomy in stages I, II, and IIIA small-cell lung cancer using the National Cancer Data Base. J Thorac Oncol 2015;10:316-23.
10. Yang $Y$, Yuan G, Zhan C, et al. Benefits of surgery in the multimodality treatment of stage IIB-IIIC small cell lung cancer. J Cancer 2019;10:5404-12.

11. Schmid S, Früh M. Immune checkpoint inhibitors and small cell lung cancer:what's new? J Thorac Dis 2018;10:S1503-8.

12. Alexandrov LB, Nik-Zainal S, Wedge DC, et al. Signatures of mutational processes in human cancer. Nature 2013;500:415-21.

13. Rudin CM, Pietanza MC, Bauer TM, et al. Rovalpituzumab tesirine, a DLL3-targeted antibody-drug conjugate, in recurrent small-cell lung cancer: a first-inhuman, first-in-class, open-label, phase 1 study. Lancet Oncol 2017;18:42-51.

14. Morgensztern D, Besse B, Greillier L, et al. Efficacy and Safety of Rovalpituzumab Tesirine in ThirdLine and Beyond Patients with DLL3-Expressing, Relapsed/Refractory Small-Cell Lung Cancer: Results From the Phase II TRINITY Study. Clin Cancer Res 2019;25:6958-66.

15. Reck M, Luft A, Szczesna A, et al. Phase III Randomized Trial of Ipilimumab Plus Etoposide and Platinum Versus Placebo Plus Etoposide and Platinum in Extensive-Stage Small-Cell Lung Cancer. J Clin Oncol 2016;34:3740-8. Erratum in: J Clin Oncol 2019;37:3327.

16. Peifer M, Fernández-Cuesta L, Sos ML, et al. Integrative genome analyses identify key somatic driver mutations of small-cell lung cancer. Nat Genet 2012;44:1104-10.

17. Beltra JC, Manne S, Abdel-Hakeem MS, et al. Developmental Relationships of Four Exhausted CD8+ T Cell Subsets Reveals Underlying Transcriptional and Epigenetic Landscape Control Mechanisms. Immunity 2020;52:825-41.e8.

18. Bustamante Alvarez JG, González-Cao M, Karachaliou N, et al. Advances in immunotherapy for treatment of lung cancer. Cancer Biol Med 2015;12:209-22.

19. Le DT, Uram JN, Wang H, et al. PD-1 Blockade in Tumors with Mismatch-Repair Deficiency. N Engl J Med 2015;372:2509-20.

20. Pardoll DM. The blockade of immune checkpoints in cancer immunotherapy. Nat Rev Cancer 2012;12:252-64.

21. Paz-Ares L, Dvorkin M, Chen Y, et al. Durvalumab plus platinum-etoposide versus platinum-etoposide in firstline treatment of extensive-stage small-cell lung cancer (CASPIAN): a randomised, controlled, open-label, phase 3 trial. Lancet Lond Engl 2019 23;394:1929-39.

22. Goldman JW, Garassino MC, Chen Y, et al. Patientreported outcomes with first-line durvalumab plus 
platinum-etoposide versus platinum-etoposide in extensive-stage small-cell lung cancer (CASPIAN): a randomized, controlled, open-label, phase III study. Lung Cancer 2020;149:46-52.

23. Wang $M$, Liang $H$, Wang $W$, et al. Immune-related adverse events of a PD-(L)1 inhibitor plus chemotherapy versus a PD-(L)1 inhibitor alone in first-line treatment for advanced non-small cell lung cancer: A meta-analysis of

Cite this article as: Xue L, Chen B, Lin J, Peng J. Anti-PD-L1 immune checkpoint inhibitors in combination with etoposide and platinum for extensive-stage small cell lung cancer: a case report. Ann Palliat Med 2021;10(1):828-835. doi: 10.21037/ apm-20-2574 randomized control trials. Cancer 2020. [Epub ahead of print]. doi: 10.1002/cncr.33270.

24. Prendergast GC, Jaffee EM. Cancer immunotherapy: immune suppression and tumor growth. Academic Press; 2013.

(English Language Editor: J. Gray) 